\title{
Factors Influencing Knowledge and Practice Regarding Cervical Cancer and Pap smear Testing among Omani Women
}

\author{
Nasar Alwahaibi*, Waad Alsalami, Nasra Alramadhani, Atheer Alzaabi
}

\begin{abstract}
Background: Knowledge of cervical cancer and performance of Pap smear testing are influenced by several sociodemographic factors. This study aimed to describe the effect of relevant variables on knowledge and compliance with guidelines in Oman. Methods: In this cross-sectional survey, participants were divided into three groups: patients who attended Outpatient Gynecology Department, female medical staff and university graduate students. Results: There were 204 outpatients, 133 staff, and 157 students. Adequate knowledge among was seen in 38.7\%, 35.3\%, and 7.6\%, respectively. Knowledge of cervical cancer and Pap smear was significantly lower among outpatients with secondary education, while those with high level of income were more likely to have adequate cancer knowledge. Uptake of Pap smear was significantly greater among outpatients aged $\geq 30$ years, with high income and a positive history of cancer. Conclusion: Culturally tailored interventions that focus on improving cancer risk knowledge are needed to maximize screening uptake for cervical cancer.
\end{abstract}

Keywords: Cervical cancer- Pap smear- knowledge- performance- sociodemographic factors

Asian Pac J Cancer Prev, 19 (12), 3367-3374

\section{Introduction}

Cervical cancer is a leading cause of morbidity and mortality among gynecological cancers worldwide, with an estimated 528,000 newly diagnosed cases in 2012 and accounting for $7.5 \%$ of all female cancer mortality (Globocan., 2016). In Oman, it accounts for 6.3\% of female cancers and ranked the third among all cancers (Nooyi and Al-Lawati., 2011). Infection with human papilloma virus (HPV), mainly 16 and 18 genotypes, is the main causative factor for cervical cancer with an estimated prevalence between 85-99\% (de Sanjose et al., 2010). Other risk factors include multiparous (Goddy et al., 2015), genetic predisposition, cigarette smoking, women's sexual activity, oral contraceptives, dietary deficiencies, and immunosuppression (Tran et al., 2015; Wang et al., 2010).

Pap smear test has a great potential for early prevention of cervical cancer. In fact, Pap test and HPV vaccine have significantly reduced the mortality and morbidity of cervical cancer (Al-Shaikh et al., 2014; Al-Darwish et al., 2014; Huh, 2010). Nevertheless, Pap smear uptake among females is still suboptimal due to poor awareness about cervical cancer and its risk factors, lack of specific knowledge regarding the procedure, physician's negative attitude, embarrassment and fear of receiving an abnormal result (Obeidat et al., 2012; Rosser et al., 2015). Indeed there is a poor awareness of HPV infection and screening tests, lack of screening guidelines and vaccine availability not only in general population but also among health professionals (Berraho et al., 2013). To the best of our knowledge, cancer risk knowledge and factors contributing to Pap smear uptake are less documented in the Middle Eastern region (Ortashi et al., 2013). Thus, this study aimed to identify and estimate the effect of contributing factors with regard to knowledge of cervical cancer and Pap smear uptake.

\section{Materials and Methods}

\section{Study design}

This cross-sectional survey was carried out from August 2015 to April 2016. Participants were Omani females from three subgroups: Outpatients from any age group who attended Outpatient Gynecology Department in Sultan Qaboos University Hospital (SQUH), staff from College of Medicine and Health Science and College of Nursing at Sultan Qaboos University (SQU), and graduating students from all the nine colleges at SQU including Agricultural and Marine Sciences, Arts and Social Sciences, Economics and Political Science, Science, Nursing, Education, Law, Engineering and Medicine and Health Sciences.

This study was ethically approved by the Medical Research Committee and Ethics Committee from the College of Medicine and Health Sciences, (SQU), Oman (MREC \# 1139). Written consent was obtained 
from all participants and they were ensured about the confidentiality and that their participation is voluntary and they have full right to withdraw from the study at any time without reasons. They were also rewarded for their time with an informative gift cards given at the end of the interview.

\section{Data Collection}

Data collection was interview-based for the gynecology outpatients and self-administered online for female staff and students. The questionnaire survey, which consists of four parts with a total of 44 questions, was designed by the investigators based on the study's objectives and literature review (Al-Darwish et al., 2014; Ortashi et al., 2013). However, some of the questions were modified based on expert opinions addressing the cultural values. Knowledge items regarding warning signs and risk factors of cervical cancer were extracted from Cancer Awareness Measure (CAM) tool kit version 2.1 and modified to fit Omani culture.

Data included age, marital status, educational level, family income, husband's educational level, family history of cancer, average visits to gynecology clinic, parity, and abortion. The parity were classified into multiparous (Para $\geq 4$ ) and non-multiparous (Para $<4$ ) according to the average number of children in Omani families. Family income (Omani Rial (OMR/Month) was divided into $\leq 1,000$ and $>1,000$ based on Oman's average monthly household income that was reported by the National Center of Statistics and Information (NSCI) (Gulf Business., 2016). The awareness questions assessed whether or not participants had ever heard of cervical cancer, HPV, HPV vaccine and Pap smear testing, knowledge of warning signs and risk factors of cervical cancer, knowledge and performance of females regarding Pap smear testing.

For each question, the answer was coded as one for correct answer and zero for incorrect or (I do not know) answer. The total score represents the sum of the correct responses of the 29 questions. Knowledge of cervical cancer and Pap smear was categorized as "adequate" if approximately $2 / 3$ (i.e. 19 ) of the 29 knowledge questions were correct. The cut-off point for knowledge adequacy was similar to other studies (Al-Shaikh et al., 2014; Jia et al., 2013).

\section{Statistical analysis}

Results of descriptive statistics were presented using Mean \pm SD for knowledge score and frequency $(\mathrm{N})$ and percentages $(\%)$ for the remaining categorical variables. Bivariate analyses were performed using $\chi^{2}$ to test the association between sociodemographic characteristics and knowledge of cervical cancer and Pap smear as well as its uptake reporting odds ratios (OR) and $95 \%$ confidence intervals (CI). P-values of less than 0.05 were considered statistically significant. The data were analyzed using Statistical Package for Social Science (SPSS) version 23 program.

\section{Results}

A total of 494 females participated in this study, 204 gynecological outpatients, 133 staff, and 157 students. Participants' characteristics were shown in Table 1. Most of participants were educated and had educational degrees. A total of $56.4 \%$ outpatients and $77.4 \%$ staff reported to have a total family income of more than 1,000 OMR per month, whereas $63.7 \%$ of participated students reported a family income of less than or equal to 1,000 OMR per month. Two-thirds of staff $(92.5 \%)$ and students $(72.6 \%)$ had not visited the clinic. Among the married females, $19.1 \%$ of the outpatients and $11.3 \%$ of staff were found to be multiparous (Para $\geq 4$ ), while $8.3 \%$ of students were non-multiparous $(\operatorname{Para}<4)$ Table 1.

\section{Awareness and knowledge of cervical cancer and Pap} smear

More than two-thirds of outpatients and students had heard of cervical cancer with less than one-third reported to have knowledge about HPV or HPV vaccine. Only $23.6 \%$ of students were aware of Pap smear testing in contrast to the high awareness among the outpatients $(61.3 \%)$ and staff $(85.7 \%)$ Table 2.

Bivariate analysis using $\chi^{2}$ showed no association between average visits to the gynecology clinic and hearing of cervical cancer, HPV, HPV vaccine and Pap smear among all participants $(\mathrm{P}>0.05)$. Only $45.1 \%$ of the outpatients and $22.9 \%$ of students knew correctly HPV infection as a risk factor for cervical cancer compared to $72.9 \%$ in staff. Using cutoff score of 19 out of 29 as an adequate knowledge score, $61.3 \%$ of the outpatients and $64.7 \%$ of staff were found to have inadequate knowledge regarding cervical cancer and Pap smear versus $92.4 \%$ in students Table 2.

Factors contributing to knowledge of cervical cancer and Pap smear

Among outpatients, marital status, educational level, and family income were associated significantly with adequate knowledge scores ( $\mathrm{P}$ 's $<0.05$ ) in contrast to age, family history of cancer, husband's educational level, parity, and abortion history $(\mathrm{P}>0.05)$. Age, marital status, family income, family history of cancer, parity and abortion were not significantly associated with knowledge scores among staff. The same was true about the association of knowledge scores with marital status, family income, and family history of cancer among students Table 3 .

A total of $36.8 \%$ and $23.3 \%$ of outpatients and staff had performed Pap smear, respectively, whereas none of students had a prior Pap smear. About 54\% of postgraduates outpatients had performed Pap smear test versus $32.2 \%$ in females at secondary level of education and $33.3 \%$ in postgraduate staff Table 4 .

Screening uptake increased with age among the outpatients (OR 1.86). Married females were four times more likely to perform Pap smear than unmarried females. Marital status was also a significant factor affecting the uptake of Pap smear test among staff. Husband's level of education was significantly correlated to Pap smear 
Table 1. Characteristics of Outpatients, Staff, and Students

\begin{tabular}{|c|c|c|c|}
\hline & $\begin{array}{c}\text { Outpatients } \\
\qquad(\mathrm{n}=204) \\
\mathrm{N}(\%)\end{array}$ & $\begin{array}{c}\text { Staff } \\
(\mathrm{n}=133) \\
\mathrm{N}(\%)\end{array}$ & $\begin{array}{c}\text { Students } \\
(\mathrm{n}=157) \\
\mathrm{N}(\%)\end{array}$ \\
\hline \multicolumn{4}{|l|}{ Age (years) } \\
\hline $20-29$ & $90(44.1)$ & $92(69.2)$ & $157(100.0)$ \\
\hline $30-39$ & $87(42.7)$ & $28(21.1)$ & $0(0.0)$ \\
\hline$\geq 40$ & $27(13.2)$ & $13(9.6)$ & $0(0.0)$ \\
\hline \multicolumn{4}{|l|}{ Marital status } \\
\hline Single & $6(2.9)$ & $56(42.1)$ & $144(91.7)$ \\
\hline Married & $198(97.1)$ & $77(57.9)$ & $13(8.3)$ \\
\hline \multicolumn{4}{|l|}{ Education } \\
\hline Below secondary education & $25(12.3)$ & $0(0.0)$ & $0(0.0)$ \\
\hline Secondary education & $59(28.9)$ & $0(0.0)$ & $0(0.0)$ \\
\hline Undergraduate & $3(1.5)$ & $37(27.8)$ & $157(100.0)$ \\
\hline Bachelor & $104(51.0)$ & $60(45.1)$ & $0(0.0)$ \\
\hline Postgraduate & $13(6.4)$ & $36(27.1)$ & $0(0.0)$ \\
\hline \multicolumn{4}{|l|}{ Husband Education* } \\
\hline Below secondary education & $21(10.6)$ & $0(0.0)$ & $0(0.0)$ \\
\hline Secondary education & $63(31.8)$ & $13(16.9)$ & $4(30.8)$ \\
\hline Undergraduate & $1(0.5)$ & $4(5.2)$ & $0(0.0)$ \\
\hline Bachelor & $92(46.5)$ & $41(53.2)$ & $8(61.5)$ \\
\hline Postgraduate & $21(10.6)$ & $19(24.7)$ & $1(7.7)$ \\
\hline \multicolumn{4}{|l|}{ Family income (OMR/Month) } \\
\hline$\leq 1000 \mathrm{OMR} /$ Month & $89(43.6)$ & $30(22.6)$ & $100(63.7)$ \\
\hline > $1000 \mathrm{OMR} /$ Month & $115(56.4)$ & $103(77.4)$ & $57(36.3)$ \\
\hline \multicolumn{4}{|l|}{ Family history of cancer } \\
\hline Negative & $117(57.4)$ & $89(61.7)$ & $89(61.7)$ \\
\hline Positive & $87(42.6)$ & $51(38.3)$ & $51(38.3)$ \\
\hline \multicolumn{4}{|l|}{ Reproductive history } \\
\hline \multicolumn{4}{|c|}{ Average visits to gynecological clinic } \\
\hline Did not visit the clinic & $0(0.0)$ & $123(92.5)$ & $114(72.6)$ \\
\hline One visit & $23(11.3)$ & $2(1.5)$ & $4(2.5)$ \\
\hline Multiple visits & $181(88.7)$ & $8(6.0)$ & $39(24.8)$ \\
\hline \multicolumn{4}{|l|}{ Parity* } \\
\hline Non-multiparous & $159(77.9)$ & $62(46.6)$ & $13(8.3)$ \\
\hline Multiparous & $39(19.1)$ & $15(11.3)$ & 0 \\
\hline \multicolumn{4}{|l|}{ Abortion* } \\
\hline No & $95(46.6)$ & $55(41.4)$ & $12(7.6)$ \\
\hline Yes & $103(50.5)$ & $22(16.5)$ & $1(0.6)$ \\
\hline
\end{tabular}

*Among married females. OMR: Omani Rial

uptake among outpatients. Outpatients who had high family income (OR 1.80) and a positive family history of cancer (OR 1.99) were more likely to perform Pap smear. While husband's level of education, income more than 1,000 OMR/Month, and a positive family history of cancer were not significantly influenced the practice of Pap smear among staff Table 5.

\section{Discussion}

Several studies suggested that adequate knowledge of cervical cancer and performance of Pap smear were influenced by several sociodemographic factors such as age, participants educational level, husband's qualification and family income (Strohl et al., 2015; Al-Shaikh et al., 2014; Al-Darwish et al., 2013; Jia et al., 2013; Obeidat et al., 2012). The findings of this study show an inadequate knowledge of Omani females with regard to cervical cancer warning signs and risk factors as well as Pap smear test. These findings are consistent with similar studies conducted on outpatients females (Barghouti et al., 2008), medical staff, e.g., nurses, doctors, and medical students 
Table 2. Awareness and Knowledge of Cervical Cancer and Pap among Participants

\begin{tabular}{|c|c|c|c|}
\hline & $\begin{array}{l}\text { Outpatients } \\
(\mathrm{n}=204)\end{array}$ & $\begin{array}{c}\text { Outpatients } \\
(\mathrm{n}=204)\end{array}$ & $\begin{array}{l}\text { Students } \\
(\mathrm{n}=157)\end{array}$ \\
\hline \multicolumn{4}{|l|}{ Awareness } \\
\hline Heard of cervical cancer & $162(79.4)$ & $130(97.7)$ & $118(75.2)$ \\
\hline Heard of HPV & $20(9.8)$ & $103(77.4)$ & $33(21.0)$ \\
\hline Heard of HPV vaccine & $12(5.9)$ & $79(59.4)$ & $23(14.6)$ \\
\hline Heard of Pap smear & $125(61.3)$ & $114(85.7)$ & $37(23.6)$ \\
\hline \multicolumn{4}{|l|}{ Early Signs and symptoms } \\
\hline Vaginal bleeding between periods & $132(64.7)$ & $86(64.7)$ & $67(42.7)$ \\
\hline Persistent lower back pain & $82(40.2)$ & $62(46.6)$ & $43(27.4)$ \\
\hline Persistent vaginal discharge with unpleasant smell & $125(61.3)$ & $74(55.6)$ & $39(24.8)$ \\
\hline Discomfort or pain during sex & $121(59.3)$ & $84(63.2)$ & $40(25.5)$ \\
\hline Menstrual periods that are longer or heavier than usual & $127(62.3)$ & $59(44.4)$ & $38(24.2)$ \\
\hline Persistent diarrhea & $118(57.8)$ & $88(66.2)$ & $65(41.4)$ \\
\hline Vaginal bleeding after menopause & $164(80.4)$ & $95(71.4)$ & $76(48.4)$ \\
\hline Persistent pelvic pain & $129(63.2)$ & $85(63.9)$ & $64(40.8)$ \\
\hline Vaginal bleeding during or after sex & $127(62.3)$ & $83(62.4)$ & $49(31.2)$ \\
\hline Blood in stool or urine & $72(35.3)$ & $21(15.8)$ & $44(28.0)$ \\
\hline Unexplained weight loss & $116(56.9)$ & $85(63.9)$ & $45(28.7)$ \\
\hline \multicolumn{4}{|l|}{ Risk factors } \\
\hline Infection with human papilloma virus & $92(45.1)$ & $97(72.9)$ & $36(22.9)$ \\
\hline Smoking & $125(61.3)$ & $82(61.7)$ & $50(31.8)$ \\
\hline Immunocompromised & $177(86.8)$ & $94(70.7)$ & $72(45.9)$ \\
\hline Immunocompromised husband & $100(49.0)$ & $37(27.8)$ & $31(19.7)$ \\
\hline Long term use of the oral contraceptive & $121(59.3)$ & $57(42.9)$ & $57(36.3)$ \\
\hline Early marriage (before 17 years) & $30(14.7)$ & $35(56.3)$ & $24(15.3)$ \\
\hline Multiparous (Para 4 and above) & $26(12.7)$ & $22(16.5)$ & $19(12.1)$ \\
\hline Family history of cervical cancer & $147(72.1)$ & $108(81.2)$ & $70(44.6)$ \\
\hline Not performing regular screening tests & $159(77.9)$ & $78(58.6)$ & $51(32.5)$ \\
\hline \multicolumn{4}{|l|}{ Pap smear } \\
\hline What is the best frequency of Pap smear? & $35(17.2)$ & $55(41.4)$ & $18(11.5)$ \\
\hline Is Pap smear required only for married females? & $149(73.0)$ & $82(61.47)$ & $100(63.7)$ \\
\hline $\begin{array}{l}\text { Is Pap smear not required for females who have reached } \\
\text { menopause? }\end{array}$ & $179(87.7)$ & $94(70.7)$ & $54(34.4)$ \\
\hline What does it mean if you have an abnormal Pap smear? & $65(31.9)$ & $65(48.9)$ & $33(21.0)$ \\
\hline \multicolumn{4}{|l|}{ After an abnormal Pap smear result: } \\
\hline Is blood test required? & $16(7.8)$ & $27(20.3)$ & $17(10.8)$ \\
\hline Is it needed to do another Pap smear? & $193(94.6)$ & $100(75.2)$ & $101(64.3)$ \\
\hline Is HPV test required? & $143(70.1)$ & $108(81.2)$ & $93(59.2)$ \\
\hline Is colposcopy needed? & $172(84.3)$ & $84(63.2)$ & $85(54.1)$ \\
\hline Is biopsy needed? & $190(93.1)$ & $108(81.2)$ & $76(49.4)$ \\
\hline Average knowledge score & $16.8 \pm 4.1$ & $16.2 \pm 5.6$ & $16.2 \pm 5.6$ \\
\hline Adequate knowledge & $79(38.7)$ & $47(35.3)$ & $47(35.3)$ \\
\hline Inadequate knowledge & $125(61.3)$ & $86(64.7)$ & $86(64.7)$ \\
\hline
\end{tabular}

(Mutyaba et al., 2006, Al-Shaikh et al., 2014).

We also found that there was a lack of knowledge about warning signs and risk factors of cervical cancer among outpatients and students. As such, more than half of females did not knew HPV infection as a risk factor which could be explained by poor knowledge about HPV infection and HPV vaccine especially in Gulf countries (Al-Darwish et al., 2014; Ortashi et al., 2013). In contrast, high awareness about HPV and its vaccine was found in other Islamic countries like Turkey 
DOI:10.31557/APJCP.2018.19.12.3367

Factors Influencing Knowledge and Practice of Cervical Cancer and Pap Smear Table 3. Factors Contributing to Knowledge of Cervical Cancer and Pap Smear

\begin{tabular}{|c|c|c|c|c|c|c|c|c|c|}
\hline \multirow[t]{4}{*}{ Characteristics } & \multicolumn{3}{|c|}{$\begin{array}{l}\text { Outpatients } \\
(\mathrm{n}=204)\end{array}$} & \multicolumn{3}{|c|}{$\begin{array}{c}\text { Staff } \\
(\mathrm{n}=133)\end{array}$} & \multicolumn{3}{|c|}{$\begin{array}{l}\text { Students } \\
(\mathrm{n}=157)\end{array}$} \\
\hline & Inadequate & Adequate & OR & Inadequate & Adequate & OR & Inadequate & Adequate & OR \\
\hline & knowledge & knowledge & & knowledge & knowledge & & knowledge & knowledge & \\
\hline & $\mathrm{N}(\%)$ & $\mathrm{N}(\%)$ & & $\mathrm{N}(\%)$ & $\mathrm{N}(\%)$ & & $\mathrm{N}(\%)$ & $\mathrm{N}(\%)$ & \\
\hline \multicolumn{10}{|l|}{ Age (years) } \\
\hline$<30^{2}$ & $56(62.2)$ & $34(37.8)$ & 1 & $55(59.8)$ & $37(40.2)$ & 1 & $145(92.4)$ & $12(7.6)$ & 3 \\
\hline$\geq 30$ & $69(60.5)$ & $45(39.5)$ & 1.07 & $31(75.6)$ & $10(24.4)$ & 0.48 & $0(0.0)$ & $0(0.0)$ & \\
\hline$P$ value & \multicolumn{2}{|c|}{0.805} & & \multicolumn{2}{|c|}{0.078} & & \multicolumn{2}{|c|}{3} & \\
\hline \multicolumn{10}{|l|}{ Marital status } \\
\hline Single $^{2}$ & $6(100.0)$ & $0(0.0)$ & & $31(55.4)$ & 25 (44.6) & 1 & $132(91.7)$ & $12(8.3)$ & 3 \\
\hline Married & $119(60.1)$ & $79(39.9)$ & 3 & $55(71.4)$ & $22(28.6)$ & 0.49 & $13(100.0)$ & $0(0.0)$ & \\
\hline$P$ value & \multicolumn{2}{|c|}{0.001} & & \multicolumn{2}{|c|}{0.308} & & \multicolumn{2}{|c|}{3} & 3 \\
\hline \multicolumn{10}{|l|}{ Education } \\
\hline Below sec educat & $21(84.0)$ & $4(16.0)$ & 0.12 & $0(0.0)$ & $0(0.0)$ & 3 & $0(0.0)$ & $0(0.0)$ & \\
\hline Secondary educat & $42(71.2)$ & $17(28.8)$ & 0.25 & $0(0.0)$ & $0(0.0)$ & 3 & $0(0.0)$ & $0(0.0)$ & \\
\hline Undergraduate & $3(100.0)$ & $0(0.0)$ & 3 & $22(59.5)$ & $15(40.5)$ & 0.96 & $145(92.4)$ & $12(7.6)$ & \\
\hline Bachelor & $54(51.9)$ & $50(48.1)$ & 0.58 & $43(71.7)$ & $17(28.3)$ & 0.55 & $0(0.0)$ & $0(0.0)$ & \\
\hline Postgraduate $^{2}$ & $5(38.5)$ & $8(61.5)$ & 1 & $21(58.3)$ & $15(41.7)$ & 1 & $0(0.0)$ & $0(0.0)$ & \\
\hline$P$ value & \multicolumn{2}{|c|}{0.001} & & \multicolumn{2}{|c|}{0.308} & \multicolumn{4}{|c|}{3} \\
\hline \multicolumn{10}{|l|}{ Husband Educa ${ }^{1}$} \\
\hline Below sec educat & $17(81.0)$ & $4(19.0)$ & 0.38 & $0(0.0)$ & $0(0.0)$ & 3 & $0(0.0)$ & $0(0.0)$ & 3 \\
\hline Secondary educat & $40(63.5)$ & $23(36.5)$ & 0.93 & $9(69.2)$ & $4(30.8)$ & 0.61 & $4(100.0)$ & $0(0.0)$ & \\
\hline Undergraduate & $1(100.0)$ & $0(0.0)$ & 3 & $2(50.0)$ & $2(50.0)$ & 1.38 & $0(0.0)$ & $0(0.0)$ & \\
\hline Bachelor & $48(52.2)$ & $44(47.8)$ & 1.49 & $33(80.5)$ & $8(19.5)$ & 0.33 & $8(100.0)$ & $0(0.0)$ & \\
\hline Postgraduate $^{2}$ & $13(61.9)$ & $8(38.1)$ & 1 & $11(57.9)$ & $8(42.1)$ & 1 & $1(100.0)$ & $0(0.0)$ & \\
\hline$P$ value & \multicolumn{2}{|c|}{0.125} & & \multicolumn{2}{|c|}{0.232} & \multicolumn{4}{|c|}{3} \\
\hline \multicolumn{10}{|c|}{ Family income (OMR/Month) } \\
\hline$\leq 1000^{2}$ & $67(75.3)$ & $22(24.7)$ & 1 & $21(70.0)$ & $9(30.0)$ & 1 & $95(95.0)$ & $5(5.0)$ & 1 \\
\hline$>1000$ & $58(50.4)$ & $57(49.6)$ & 2.99 & $65(63.1)$ & $38(36.9)$ & 1.36 & $50(87.7)$ & $7(12.3)$ & 2.66 \\
\hline$P$ value & \multicolumn{2}{|c|}{$<0.001$} & & \multicolumn{2}{|c|}{0.487} & & & & \\
\hline Family history of canc & & & & & & & & & \\
\hline Negative $^{2}$ & $74(63.2)$ & $43(36.8)$ & 1 & $52(63.4)$ & $30(36.6)$ & 1 & $101(91.8)$ & $9(8.2)$ & 1 \\
\hline Positive & $51(58.6)$ & $36(41.4)$ & 1.22 & $34(66.7)$ & $17(33.3)$ & 0.86 & $44(93.6)$ & $3(6.4)$ & 0.77 \\
\hline$P$ value & & & & & & & & & \\
\hline Parity ${ }^{1}$ & & & & & & & & & \\
\hline Non-multiparous ${ }^{2}$ & $93(58.5)$ & $66(41.5)$ & 1 & $44(71.0)$ & $18(29 / 0)$ & 1 & $0(0.0)$ & $0(0.0)$ & 3 \\
\hline Multiparous & $26(66.7)$ & $13(33.3)$ & 0.71 & $11(73.3)$ & $4(26.7)$ & 0.88 & $0(0.0)$ & $13(100.0)$ & \\
\hline P value & & & & & & & & & \\
\hline Abortion1 & & & & & & & & & \\
\hline $\mathrm{No}^{2}$ & $59(62.1)$ & $36(37.9)$ & 1 & $38(69.1)$ & $17(30.9)$ & 1 & $0(0.0)$ & $12(100.0)$ & 3 \\
\hline Yes & $60(58.3)$ & $43(41.7)$ & 1.18 & $17(77.3)$ & $5(22.7)$ & 0.66 & $0(0.0)$ & $1(100.0)$ & \\
\hline$P$ value & 0.580 & & & 0.473 & & & 3 & & \\
\hline
\end{tabular}

${ }^{1}$ Only among married females, ${ }^{2}$ Reference category, ${ }^{3}$ P-value, OR and $95 \%$ CI cannot be calculated OMR: Omani Rial, se: secondary, educat: education, OR: Odds ratios

$(45.0-46.0 \%)$ (Dursun et al., 2009) and non-Islamic countries like Belgium (50.0\%) (Donders et al., 2008). This might be due to conservative society in Gulf countries and lack of public program for sexual and reproductive education. It was observed that countries that provide good sexual and reproductive education program like Australia showed high levels of knowledge (80.0-90.0\%) (Giles and Garland., 2006). We found that knowledge of HPV as etiological factor of cervical cancer was high among our medical staff $(72.9 \%)$, in contrast to data from Jordan which showed almost half of health professionals did not knew about HPV infection as a risk factor (Obeidat et al., 2012).

We assessed the influence of some independent variables on knowledge level of cervical cancer and Pap smear. Educational level and family income were significant independent predictors of knowledge level among outpatients, comparable to previous studies 
Table 4. Factors Contributing to Knowledge of Pap smear

\begin{tabular}{|c|c|c|c|c|c|c|}
\hline \multirow[t]{2}{*}{ Characteristics } & \multicolumn{2}{|c|}{ Outpatients ( $\mathrm{n}=204)$} & \multicolumn{2}{|c|}{ Staff $(n=133)$} & \multicolumn{2}{|c|}{ Students $(\mathrm{n}=157)$} \\
\hline & $\begin{array}{c}\text { Performed } \\
\text { N (\%) }\end{array}$ & $\begin{array}{c}\text { Not Performed } \\
\text { N }(\%)\end{array}$ & $\begin{array}{c}\text { Performed } \\
\text { N (\%) }\end{array}$ & $\begin{array}{c}\text { Not Performed } \\
\text { N }(\%)\end{array}$ & $\begin{array}{c}\text { Performed } \\
\text { N (\%) }\end{array}$ & $\begin{array}{c}\text { Not Performed } \\
\text { N (\%) }\end{array}$ \\
\hline \multicolumn{7}{|l|}{ Age (years) } \\
\hline$<30^{2}$ & $26(28.9)$ & $64(71.1)$ & $18(19.6)$ & $74(80.4)$ & $0(0.0)$ & $157(100.0)$ \\
\hline$\geq 30$ & $49(43.0)$ & $65(57.0)$ & $13(31.7)$ & $28(68.3)$ & $0(0.0)$ & $0(0.0)$ \\
\hline P value & \multicolumn{2}{|c|}{0.038} & \multicolumn{2}{|c|}{0.126} & \multicolumn{2}{|c|}{3} \\
\hline \multicolumn{7}{|l|}{ Marital status } \\
\hline Single $^{2}$ & $0(0.0)$ & $6(100.0)$ & $6(10.7)$ & $50(89.3)$ & $0(0.0)$ & $144(100.0)$ \\
\hline Married & 75 (37.9) & $123(62.1)$ & $25(32.5)$ & $52(67.5)$ & $0(0.0)$ & $13(100.0)$ \\
\hline P value & \multicolumn{2}{|c|}{0.050} & \multicolumn{2}{|c|}{0.003} & \multicolumn{2}{|c|}{3} \\
\hline \multicolumn{7}{|l|}{ Education } \\
\hline Below sec educat & $10(40.0)$ & $15(60.0)$ & $0(0.0)$ & $0(0.0)$ & $0(0.0)$ & $0(0.0)$ \\
\hline Secondary educat & $19(32.2)$ & $40(67.8)$ & $0(0.0)$ & $0(0.0)$ & $0(0.0)$ & $0(0.0)$ \\
\hline Undergraduate & $0(0.0)$ & $3(100.0)$ & $5(13.5)$ & $32(86.5)$ & $0(0.0)$ & $157(0.0)$ \\
\hline Bachelor & $39(37.5)$ & $65(62.5)$ & $14(23.3)$ & $46(76.7)$ & $0(0.0)$ & $0(0.0)$ \\
\hline Postgraduate $^{2}$ & $7(53.8)$ & $6(46.2)$ & $12(33.3)$ & $24(66.7)$ & $0(0.0)$ & $0(0.0)$ \\
\hline $\mathrm{P}$ value & \multicolumn{2}{|c|}{0.401} & \multicolumn{2}{|c|}{0.135} & \multicolumn{2}{|c|}{3} \\
\hline \multicolumn{7}{|l|}{ Husband Educa ${ }^{1}$} \\
\hline Below sec educat & $7(33.3)$ & $14(66.7)$ & $0(0.0)$ & $0(0.0)$ & $0(0.0)$ & $0(0.0)$ \\
\hline Secondary educat & $21(33.3)$ & $42(66.7)$ & $3(23.1)$ & $10(76.9)$ & $0(0.0)$ & $4(100.0)$ \\
\hline Undergraduate & $0(0.0)$ & $1(100.0)$ & $3(75.0)$ & $1(25.0)$ & $0(0.0)$ & $0(0.0)$ \\
\hline Bachelor & $33(35.9)$ & $59(64.1)$ & $10(24.4)$ & $31(75.6)$ & $0(0.0)$ & $8(100.0)$ \\
\hline Postgraduate $^{2}$ & $14(66.7)$ & $7(33.3)$ & $9(47.4)$ & $10(52.6)$ & $0(0.0)$ & $1(100.0)$ \\
\hline$P$ value & \multicolumn{2}{|c|}{0.062} & \multicolumn{2}{|c|}{0.083} & \multicolumn{2}{|c|}{3} \\
\hline \multicolumn{7}{|c|}{ Family income (OMR/Month) } \\
\hline$\leq 1000^{2}$ & $26(29.2)$ & $63(70.8)$ & $0(0.0)$ & $30(100.0)$ & $0(0.0)$ & $100(100.0)$ \\
\hline$>1000$ & $49(42.6)$ & $66(57.4)$ & $31(30.1)$ & $72(69.9)$ & $0(0.0)$ & $57(100.0)$ \\
\hline $\mathrm{P}$ value & \multicolumn{2}{|c|}{0.049} & \multicolumn{2}{|c|}{0.001} & \multicolumn{2}{|c|}{3} \\
\hline \multicolumn{7}{|c|}{ Family history of cancer } \\
\hline Negative $^{2}$ & $35(29.9)$ & $82(70.1)$ & $21(25.6)$ & $61(74.4)$ & $0(0.0)$ & $110(100.0)$ \\
\hline Positive & $40(46.0)$ & $47(54.0)$ & $10(19.6)$ & $41(80.4)$ & $0(0.0)$ & $47(100.0)$ \\
\hline $\mathrm{P}$ value & \multicolumn{2}{|c|}{0.019} & & & & 3 \\
\hline Parity $^{1}$ & & & & & & \\
\hline Non-multiparous ${ }^{2}$ & $56(35.2)$ & $103(64.8)$ & $20(32.3)$ & $42(67.7)$ & $0(0.0)$ & $13(100.0)$ \\
\hline Multiparous & $19(48.7)$ & $20(51.3)$ & $5(33.3)$ & $10(66.7)$ & $0(0.0)$ & $0(0.0)$ \\
\hline $\mathrm{P}$ value & & & & & & 3 \\
\hline Abortion $^{1}$ & & & & & & \\
\hline $\mathrm{No}^{2}$ & $30(31.6)$ & $65(68.4)$ & $8(36.4)$ & $38(69.1)$ & $0(0.0)$ & $12(100.0)$ \\
\hline Yes & $45(43.7)$ & $58(56.3)$ & $17(30.9)$ & $14(63.6)$ & $0(0.0)$ & $1(100.0)$ \\
\hline$P$ value & & & & & & 3 \\
\hline
\end{tabular}

${ }^{1}$ Only among married females, ${ }^{2}$ Reference category, ${ }^{3}$ P-value, OR and 95\% CI cannot be calculated OMR: Omani Rial, se: secondary, educat: education

(Barghouti et al., 2008; Al Sairafi and Mohamed, 2009; Al-Meer at al., 2011). Also, outpatients with higher family income had better cancer knowledge scores as confirmed by other series (Strohl et al., 2015). High level of income might have influenced cancer knowledge level directly and indirectly by providing more chances for receiving better education and highly standard healthcare where health professionals have more time to raise the knowledge level regarding important health issues.

It is noteworthy that, educational level and family income of medical staff did not influence their level of knowledge. This finding is in line with other similar studies (Obeidat et al., 2012; Mutyaba et al., 2006). Health care providers and all of the medical staff are expected to be adequately knowledgeable about all the aspects related to cervical cancer and Pap smear because they play an active role in the provision of appropriate information about the warning signs, risk factors and importance of screening test to their patients and the public.

Most of students had inadequately knowledge about cervical cancer and screening. Similar finding was found in a study conducted on medical students in Saudi Arabia 
Table 5. Factors Associated with Uptake of Pap smear among Outpatients and Staff

\begin{tabular}{|c|c|c|}
\hline & Outpatients $(\mathrm{n}=204)$ OR $(95 \% \mathrm{CI})$ & Staff $(n=133)$ OR $(95 \% C I)$ \\
\hline \multicolumn{3}{|l|}{ Age } \\
\hline$<30 \mathrm{yrs}^{1}$ & 1 & 1 \\
\hline$\geq 30 \mathrm{yrs}$ & $1.86(1.03-3.34)$ & $1.91(0.83-4.40)$ \\
\hline \multicolumn{3}{|l|}{ Marital status } \\
\hline Single $^{1}$ & 1 & 1 \\
\hline Married & $0.95(0.92-0.99)$ & $4.00(1.52-10.59)$ \\
\hline \multicolumn{3}{|l|}{ Education } \\
\hline Below secondary education & $0.57(0.15-2.21)$ & -2 \\
\hline Secondary education & $0.41(0.12-1.38)$ & -2 \\
\hline Undergraduate & -2 & $0.31(0.10-1.01)$ \\
\hline Bachelor & $0.51(0.16-1.64)$ & $0.61(0.24-1.5)$ \\
\hline Postgraduate $^{1}$ & 1 & 1 \\
\hline \multicolumn{3}{|l|}{ Husband Education } \\
\hline Below secondary education & $0.25(0.07-0.90)$ & -2 \\
\hline Secondary education & $0.25(0.09-0.71)$ & $0.33(0.07-1.61)$ \\
\hline Undergraduate & -2 & $3.33(0.29-38.09)$ \\
\hline Bachelor & $0.28(0.10-0.76)$ & $0.36(0.11-1.13)$ \\
\hline Postgraduate $^{1}$ & 1 & 1 \\
\hline \multicolumn{3}{|l|}{ Family income (OMR/Month) } \\
\hline$\leq 1000^{1}$ & 1 & -2 \\
\hline$>1000$ & $1.80(1.11-3.24)$ & \\
\hline \multicolumn{3}{|l|}{ Family history of cancer } \\
\hline Negative $^{1}$ & 1 & 1 \\
\hline Positive & $1.99(1.12-3.56)$ & $0.71(.30-1.66)$ \\
\hline \multicolumn{3}{|l|}{ Parity } \\
\hline Non-multiparous ${ }^{1}$ & 1 & 1 \\
\hline Multiparous & $1.75(0.86-3.54)$ & $1.05(0.32-3.48)$ \\
\hline \multicolumn{3}{|l|}{ Abortion } \\
\hline $\mathrm{No}^{1}$ & 1 & 1 \\
\hline Yes & $1.68(0.94-3.01)$ & $1.28(0.45-3.61)$ \\
\hline \multicolumn{3}{|c|}{ Knowledge level of cervical cancer and Pap smear } \\
\hline Inadequate $^{1}$ & 1 & 1 \\
\hline Adequate & $1.55(0.87-2.77)$ & $1.01(0.44-2.34)$ \\
\hline
\end{tabular}

${ }^{1}$ Reference category, ${ }^{2} \mathrm{OR}$ cannot be calculated, OMR: Omani Rial, OR: Odds ratios.

(Al-Shaikh et al., 2014). Our data suggests that this subgroup as a future new generation needs to be highly educated about important health issues and screening programs.

Marital status influenced significantly the level of knowledge among outpatients. This result was similar to a study conducted in Michigan (Holcomb et al., 2004). However, age and husband education were not associated with the knowledge score among females participated in the study, consistent with other similar study (Su., 2011). In addition, the findings of this study show that parity and abortion were not associated with knowledge scores among the outpatients, staff and students, in disagreement with other studies (Al-Meer et al., 2011; Su, 2011).

Among outpatients, $36.8 \%$ had undergone a Pap smear test. This, in consistent with other studies, suggests a low uptake among females possibly due to lack of national preventive health-screening program, lack of encouragement by health professionals (Barghouti et al., 2008; Al-Meer at al., 2011; Ranabhat et al., 2014) as only $32.4 \%$ of females were recommended by health professionals to do Pap smear. In contrary, in Jordan, most of the Pap smear testing among female outpatients was encouraged by health providers (Obeidat et al., 2012).

Outpatients were more likely to perform Pap smear if they were older than 30 years, had a family income $>1,000 \mathrm{OMR} /$ Month, and had a positive family history of cancer. This finding is in line with other similar studies (Barghouti et al., 2008; Al-Meer at al., 2011). This might be due to more access to health care facilities and performing regular check-ups.

As such, female outpatients who are married to a husband with secondary education or below were less likely to perform Pap smear. Although this finding is in disagreement other study ( $\mathrm{Su}, 2011)$, education is considered as an important predictor of performance and 
knowledge of Pap smear (Barghouti et al., 2008; Su, 2011).

Married female staff were more likely to perform Pap smear test than single females. This would be explained that married females are more likely to have multiple visits to gynecology clinic for their contraceptive and obstetric consultation. Unlike other study conducted among nurses (Tay et al., 2015), the present study showed that husband education is not an influencing factor on the practice of Pap smear among female staff. While, many of the demographic factors such as age, family income, family history of cancer, parity, and abortion were found to correlate positively with uptake of Pap smear test among the medical staff in previous study (Ackerson and Gretebeck, 2007), this correlation was not recorded in the present study.

In conclusion, the findings of this study showed inadequate knowledge of cervical cancer and poor uptake of Pap smear test among outpatients, staff, and medical students. As a recommendation, culturally appropriate educational health programs and interventions that focus on improving the level of knowledge are needed to maximize screening uptake with Pap smear testing.

\section{References}

Ackerson K, Gretebeck K (2007). Factors influencing cancer screening practices of underserved women. J Am Acad Nurse Pract, 19, 591-6.

Al Sairafi M, Mohamed FA (2009). Knowledge, attitudes, and practice related to cervical cancer screening among Kuwaiti women. Med Princ Pract, 18, 35-42.

Al-Darwish AA, Al-Naim AF, Al-Mulhim KS, et al (2014). Knowledge about cervical cancer early warning signs and symptoms, risk factors and vaccination among students at a medical school in Al-Ahsa, Kingdom of Saudi Arabia. Asian Pac J Cancer Prev, 15, 2529-32.

Al-Meer FM, Aseel MT, Al-Khalaf J, Al-Kuwari MG, Ismail MF (2011). Knowledge, attitude and practices regarding cervical cancer and screening among women visiting primary health care in Qatar. East Mediterr Health J, 17, 855-61.

Al-Shaikh GK, Almussaed EM, Fayed AA, et al (2014). Knowledge of Saudi female university students regarding cervical cancer and acceptance of the human papilloma virus vaccine. Saudi Med J, 35, 1223-30.

Barghouti FF, Takruri AH, Froelicher ES (2008). Awareness and behavior about Pap smear testing in family medicine practice. Saudi Med J, 29, 1036-40.

Berraho M, Fakir SE, Abda N, Mathoulin-Pelissier S, Nejjari C (2013). HPV and cervical cancer: knowledge and practices of physicians in Fez. Sante Publique, 25, 351-7.

de Sanjose S, Quint WG, Alemany L, et al (2010). Human papillomavirus genotype attribution in invasive cervical cancer: a retrospective cross-sectional worldwide study. Lancet Oncol, 11, 1048-56.

Donders GG, Gabrovska M, Bellen G, et al (2008). Knowledge of cervix cancer, human papilloma virus (HPV) and HPV vaccination at the moment of introduction of the vaccine in women in Belgium. Arch Gynecol Obstet, 277, 291-8.

Dursun P, Altuntas B, Kuscu E, Ayhan A (2009). Women's knowledge about human papillomavirus and their acceptance of HPV vaccine. Aust N Z J Obstet Gynaecol, 49, 202-6.

Giles M, Garland S (2006). A study of women's knowledge regarding human papillomavirus infection, cervical cancer and human papillomavirus vaccines. Aust $N Z$ J Obstet Gynaecol, 46, 311-5.
Globocan.iarc.fr. (2016). GLOBOCAN Cancer Fact Sheets: Cervical cancer. [online] Available at: http://globocan.iarc.fr/ old/FactSheets/cancers/cervix-new.asp [cited April 4, 2016].

Goddy B, Kennedy N, Michael O (2015). Profile and retrospective analysis of the use of preventive strategies in patients with cervical cancer in South-South Nigeria. Niger J Med, 56, 109-12.

Gulf Business (2016). Oman's Household Income Rises 83.9\% In A Decade. Gulf Business .[online] Available at: http:// www.gulfbusiness.com/articles/industry/omans-householdincome-rises-83-9-in-a-decade/ [cited April 26, 2017].

Holcomb B, Bailey JM, Crawford K, Ruffin MT (2004). Adults' knowledge and behaviors related to human papillomavirus infection. J Am Board Fam Pract, 17, 26-31.

Huh WK (2010). The changing face of cervical cancer screening in the United States. JNCCN, 8, 1329-30.

Jia Y, Li S, Yang R, et al (2013). Knowledge about cervical cancer and barriers of screening program among women in Wufeng county, a high-incidence region of cervical cancer in China. PLoS One, 8, e67005.

Mutyaba T, Mmiro F, Weiderpass E (2006). Knowledge, attitudes, and practices on cervical cancer screening among the medical workers of Mulango Hospital, Uganda. BMC Med Educ, 6, 13-9.

Nooyi SC, Al-Lawati JA (2011). Cancer incidence in Oman, 1998-2006. Asian Pac J Cancer Prev, 12, 1735-8.

Obeidat BR, Amarin ZO, Alzaghal L (2012). Awareness, practice and attitude to cervical Papanicolaou smear among female health care workers in Jordan. Eur J Cancer Care, 21, 372-6.

Ortashi O, Raheel H, Shalal M, Osman N (2013). Awareness and knowledge about human papillomavirus infection and vaccination among women in UAE. Asian Pac J Cancer Prev, 1, 6077-80.

Ranabhat S, Tiwari M, Dhungana G, Shrestha R (2014). Association of knowledge, attitude and demographic variables with cervical Pap smear practice in Nepal. Asian Pac J Cancer Prev, 15, 8905-10.

Rosser JI, Njoroge B, Huchko MJ (2015). Knowledge about cervical cancer screening and perception of risk among women attending outpatient clinics in rural Kenya. Int $J$ Gynaecol Obstet, 128, 211-5.

Strohl A, Mendoza G, Ghant M, et al (2015). Barriers to prevention: knowledge of HPV, cervical cancer, and HPV vaccinations among African American women. Am JObstet Gynecol, 212, 65.e1-e5.

Su TT (2011). Factors related to poor practice of pap smear screening among secondary school teachers in Malaysia. Asian Pac J Cancer Prev, 12, 1347-52.

Tay K, Tay SK, Tesalona KC, et al (2015). Factors affecting the uptake of cervical cancer screening among nurses in Singapore. Int J Gynaecol Obstet, 130, 230-4.

Tran LT, Tran LT, Bui TC, et al (2015) Risk factors for high-risk and multi-type Human Papillomavirus infections among women in Ho Chi Minh City, Vietnam:A cross-sectional study. BMC Womens Health, 15, 172-7.

Wang SS, Gonzalez P, Yu K, et al (2010). Common genetic variants and risk for HPV persistence and progression to cervical cancer. PLoS One, 5, e8667.

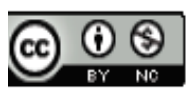

This work is licensed under a Creative Commons AttributionNon Commercial 4.0 International License. 